\title{
Tailoring basemaps for color vision impaired people
}

\author{
J. M. Misiukas ${ }^{\text {a }}$, V. van Duijnhoven ${ }^{\mathrm{a}}$, R. A. Klöpping ${ }^{\mathrm{a}}$, G. Porras Cantons ${ }^{\mathrm{a}}$ \\ jennifer.misiukas@gmail.com, vincent@ vanduijnhoven.xyz, rick.klopping@ outlook.com, gerardporras@ gmail.com
}

${ }^{\text {a }}$ Master Geo-Information Science, Wageningen University \& Research, Wageningen, The Netherlands

\begin{abstract}
Widely used basemaps are not particularly inclusive when it comes to color vision impaired users. The use of colors, even though perfect for the representation of features, does not always account for color vision impairments. Certain combinations of colors in basemaps are indistinguishable for colorblind people. With the awareness that those disabilities should be considered when designing a basemap, Esri Nederland and Kadaster instigated the research on the topic, aiming to create a methodology on how to optimize the basemaps for color vision impaired users. The methodology developed was applied in the assessment of six basemaps and improvements were performed in the two most commonly used basemaps in the Netherlands, the Esri World Topographic Map, and the BRT Achtergrondkaart Standaard. Suitable shades were selected to replace the problematic ones in the original basemaps, and the target group evaluated the improvements through surveys. The results showed that the commonly used basemaps are able to well represent the features in a colorblind-friendly way. However, there is room for improvements in order to achieve more suitable and inclusive basemaps.
\end{abstract}

Keywords: Basemaps, Color vision impairment, Vector tiles, Topographic maps

\section{Introduction}

Topographic maps are two-dimensional representations of natural and artificial features found on the planet (Kadaster, 2020). Topographic elements help to identify the main area's characteristics, so they are often used as basemaps. The majority of these maps however are created with little or no consideration to the color vision impaired users (Hobbins, 2019). A problem for color vision impaired people is that they see the colors differently. As a result, map features in basemaps can be harder to distinguish. Basemaps are purposely muted background maps that are used as a foundation for the information that will be represented. Therefore, they do not allow for the use of strategic elements such as hatches or specific symbology that would make them more readable for color vision impaired users. On average, $8 \%$ of men and $0,5 \%$ of women experience a type of color vision impairment (Colblindor, 2010). There are various types of color vision impairment, which can be divided into three categories namely: Monochromatism (total color blindness in which the vision is reduced to seeing black, white, and grey), Dichromatism (blindness for one color), or Anomalous Trichromatism (a weakness for a certain color). Monochromatism can be further specified into Achromatopsia (rod-monochromatism), which means that the vision is deeply or entirely impaired, being decreased to seeing just the intensity of light emanating from an object. Dichromatism and Trichromatism can be further divided into blindness or weakness for either the color red (protanopia and protanomaly), green (deuteranopia and deuteranomaly), or blue (tritanopia and tritanomaly) (Wong, 2011). The red and green color vision impairments are the most common, while the blue impairment and the full color blindness are rare. The red and green color vision impairments are remarkably similar as can be seen in the simulation represented in Figure 1. The figure shows how a color scheme can be optimized for color vision impaired people.

In collaboration with Esri Nederland and Kadaster, research was conducted into how to optimize the basemaps for color vision impaired people. Based on previous research on the optimization of maps, simply avoiding certain combinations of colors is not enough to create a colorblind-friendly design (Harrower \& Brewer, 2013). Currently, there are available tools that support the creation of optimized maps, beside the possibility of using specific palettes or increasing the contrast between certain features on the map. However, basemaps depend on a well-developed order for their features, and adding a new set of rules for colorblind optimization can be somewhat discouraging (Skinner, 2020). The goal was, therefore, to create a methodology that could be applied on basemaps in order to optimize them for color vision impairments, 


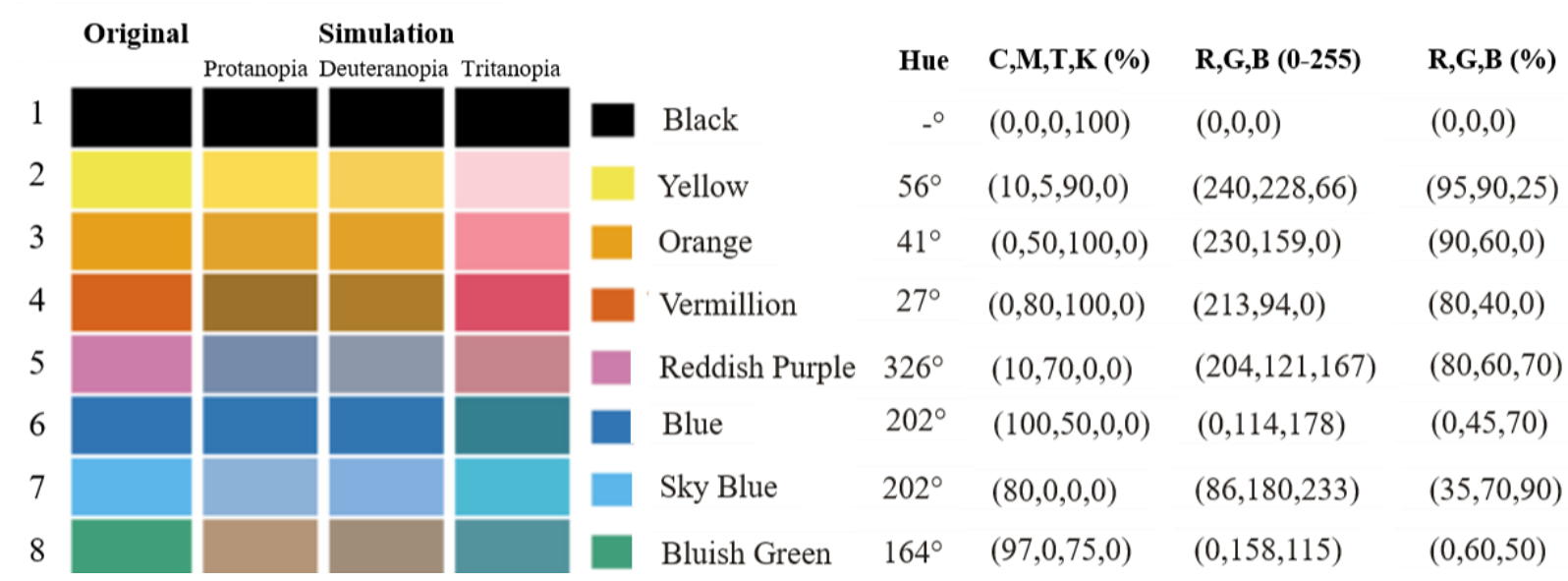

Figure 1. Suggestion for a colorblind-safe palette. The first column indicates the original color, and the next three columns indicate simulations for each type of color vision impairment (adapted from (Okabe \& Ito, 2002)).

solving the question of How can the accessibility of basemaps for colorblind people be improved? By optimizing their basemaps, Esri Nederland and Kadaster can, as geodata providers, make maps more accessible to colorblind users.

\section{Methods}

The following methodology was created to optimize basemaps for color vision impairments.

\section{Current state assessment}

The purpose of the current state assessment (CSA) was to analyze how suitable current basemaps are for color vision impaired people and where there is room for improvement. The CSA consisted of two steps which were applied to a selection of the most common basemaps used in the Netherlands (BRT achtergrondkaart Standaard, BRT achtergrondkaart Grijs, Esri World Topographic Map, Esri Light Gray Canvas, Google Maps, and OpenStreetMap).

First, all the colors visibly used in a basemap were extracted using an eyedropper tool. Next, these colors were combined based on color combinations that are known to be a problem for people with color vision impairments. The literature study provided the following problematic color combinations (Colblindor, 2009): green \& red, green \& brown, blue \& purple, green \& blue, light green \& yellow, red, \& yellow, green \& gray, and green \& black. At last, using the tool Color Oracle (Color Oracle, 2018), the different types of color vision impairments were simulated. This demonstrated if two colors were conflicting or if the colors were still distinguishable enough when next to each other.
The second step of the CSA was to check the distinguishability of different topographic features. The use of certain colors to represent those features might cause them to become unrecognizable by color impaired users of the basemaps.

Table 1. Assessment plan developed to evaluate the selected basemaps where: P=protanopia, $\mathrm{D}=$ deuteranopia, $\mathrm{T}=$ tritanopia, and $\mathrm{A}=$ achromatopsia.

\begin{tabular}{|c|c|c|c|c|c|c|}
\hline & & \multirow{2}{*}{ Weight } & \multicolumn{4}{|c|}{ Basemap } \\
\hline & & & $\mathrm{P}$ & D & $\mathrm{T}$ & A \\
\hline \multirow{5}{*}{$\begin{array}{l}\text { Topographic } \\
\text { Elements }\end{array}$} & Ad. boundaries & 5 & & & & \\
\hline & Building types & 1 & & & & \\
\hline & Landuse & 4 & & & & \\
\hline & Road types & 3 & & & & \\
\hline & Railway & 2 & & & & \\
\hline \multirow{2}{*}{ Symbology Use } & Dashed lines & 1 & & & & \\
\hline & Texture & 1 & & & & \\
\hline Typography & Contrast & 1 & & & & \\
\hline \multicolumn{2}{|r|}{ Total } & 18 & & & & \\
\hline
\end{tabular}

The information observed with the colorblind simulator was combined into an assessment plan (Table 1). Using a rating system, points were assigned to the selected features, and that resulted in a final percentage that indicated how suitable a map currently is for users with color vision impairments. The rating system classified each feature from -2 to 2 indicating suitability (or lack of thereof). The rate of 2 indicates a distinguishable feature, while a -2 suggests that many features are indistinguishable. For the symbology use, a 2 indicates that either the use of dashed lines or texture was made to enhance distinguishability where needed. The 
assessment also provided an overview of elements that were believed to be challenging for color vision impaired people.

\section{Surveys among color vision impaired users}

As described, the method was performed by the authors (non-color vision impaired people). Using surveys, the outcomes of the CSA were verified among a group of color vision impaired people. Two surveys were developed in May/June of 2020 and had the participation of 27 color vision impaired users in the initial survey that included the CSA and preliminary improvements and 19 participants in a survey with the second round of improvements.

\section{Improvement of the basemaps}

Based on the CSA and feedback from the users with color vision impairments, the basemaps could be improved. To make quick style variations on the basemaps, the vector tile technology was applied (Esri, 2015). Color vision impairments for red or green are quite similar to each other and often, a single style can be made to cover both forms of color vision impairments. Because a color vision impairment for blue is significantly different from the others, a different map style is often needed. After some style variations were made, these were checked by a group of color vision impaired people $(n=27)$. The feedback was then used for the second round of improvements which was verified again by the target group $(n=19)$. The improved basemaps were also checked by noncolorblind people $(n=3)$ to check whether the improvements on the basemaps were appreciated or if the current basemaps are preferred.

\subsection{Case study}

The depicted method was tested on two basemaps, namely the World Topographic Map by Esri and the BRT Achtergrondkaart Standaard by Kadaster. The basemap by Esri was already available as a vector tile, but the vector data map from Kadaster had to be first created from the raw topographic data.

As a final step, an example map was made where data was overlayed on the optimized basemaps. The overlay data was also optimized for color vision impairments to test the color interaction and distinguishability of overlay data with the basemaps. This map was also evaluated by the user group in the survey performed after the second round of improvements.

\subsection{Software and Data Availability}

For this research, several software, tools, and services were used. To simulate color vision impairment, the freely available tool Color Oracle (Color Oracle, 2018)

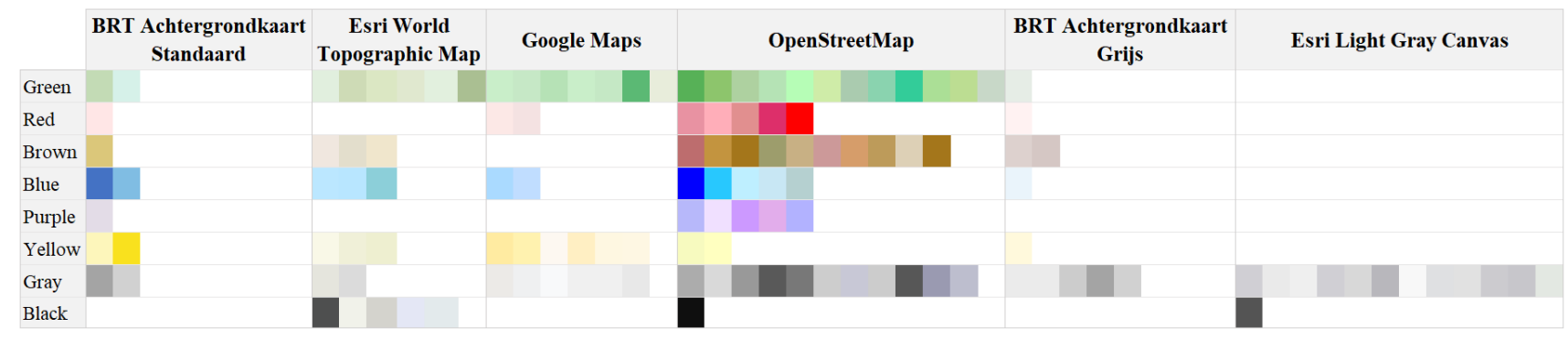

Figure 2. Color palettes collected from the assessed basemaps using an eyedropper tool.

Table 2. Current state assessment of basemap features $(P=$ protanopia, $D=$ deuteranopia, $T=$ tritanopia, $A=$ achromatopsia $)$.

\begin{tabular}{|c|c|c|c|c|c|c|c|c|c|c|c|c|c|c|c|c|c|c|c|c|c|c|c|c|c|c|}
\hline & & \multirow[t]{2}{*}{ Weight } & \multicolumn{4}{|c|}{ BRT Standaard } & \multicolumn{4}{|c|}{$\begin{array}{c}\text { Esri World } \\
\text { Topographic Map }\end{array}$} & \multicolumn{4}{|c|}{ Google Maps } & \multicolumn{4}{|c|}{ OpenStreetMaps } & \multicolumn{4}{|c|}{ BRT Grijs } & \multicolumn{4}{|c|}{$\begin{array}{c}\text { Esri Light Gray } \\
\text { Canvas }\end{array}$} \\
\hline & & & $\mathrm{P}$ & $\mathrm{D}$ & $\mathrm{T}$ & A & $\mathrm{P}$ & $\mathrm{D}$ & $\mathrm{T}$ & $\mathrm{A}$ & $\mathrm{P}$ & $\mathrm{D}$ & $\mathrm{T}$ & A & $\mathrm{P}$ & $\mathrm{D}$ & $\mathrm{T}$ & A & $P$ & $\mathrm{D}$ & $\mathrm{T}$ & A & $\mathrm{P}$ & $\mathrm{D}$ & $\mathrm{T}$ & A \\
\hline \multirow{5}{*}{$\begin{array}{l}\text { Topographic } \\
\text { Elements }\end{array}$} & Ad. boundaries & 5 & 2 & 2 & 2 & 2 & 2 & 2 & 2 & 2 & 2 & 2 & 2 & 2 & 1 & 1 & 1 & 1 & 2 & 2 & 2 & 2 & 2 & 2 & 2 & 2 \\
\hline & Building types & 1 & 2 & 2 & 2 & 2 & 1 & 1 & 1 & 1 & 2 & 1 & 2 & 1 & 2 & 2 & 2 & 2 & 2 & 2 & 2 & 2 & 2 & 2 & 2 & 2 \\
\hline & Landuse & 4 & 2 & 2 & 1 & 0 & 1 & 1 & 2 & 0 & 1 & 1 & 2 & 0 & 0 & 0 & 2 & 0 & 1 & 1 & 0 & -2 & 2 & 2 & 2 & 2 \\
\hline & Road types & 3 & 2 & 2 & 2 & 2 & 2 & 2 & 2 & 2 & 2 & 2 & 2 & 2 & 2 & 2 & 2 & 1 & 2 & 2 & 2 & 2 & 2 & 2 & 2 & 2 \\
\hline & Railway & 2 & -2 & -2 & -2 & -2 & 2 & 2 & 2 & 2 & -2 & -2 & -2 & -2 & 2 & 2 & 2 & 2 & 2 & 2 & 2 & 2 & 0 & 0 & 0 & 0 \\
\hline \multirow{2}{*}{ Symbology Use } & Dashed lines & 1 & 2 & 2 & 2 & 2 & 2 & 2 & 2 & 2 & 2 & 2 & 2 & 2 & 1 & 1 & 1 & 1 & 2 & 2 & 2 & 2 & 2 & 2 & 2 & 2 \\
\hline & Texture & 1 & 0 & 0 & 0 & 0 & -1 & -1 & -1 & -1 & 0 & 0 & 0 & 0 & 2 & 2 & 2 & 2 & 0 & 0 & 0 & 0 & 0 & 0 & 0 & 0 \\
\hline \multirow[t]{2}{*}{ Typography } & Contrast & 1 & 2 & 2 & 2 & 2 & 2 & 2 & 2 & 2 & 2 & 2 & 2 & 2 & 2 & 2 & 2 & 2 & 2 & 2 & 2 & 2 & 2 & 2 & 2 & 2 \\
\hline & Total & 18 & 1.4 & 1.4 & 1.2 & 1.0 & 1.6 & 1.6 & 1.8 & 1.3 & 1.2 & 1.2 & 1.4 & 0.9 & 1.2 & 1.2 & 1.7 & 1.1 & 1.7 & 1.7 & 1.4 & 1.0 & 1.7 & 1.7 & 1.7 & 1.7 \\
\hline \multicolumn{3}{|c|}{ Suitability for color vision impaired users } & \multicolumn{4}{|c|}{$82 \%$} & \multicolumn{4}{|c|}{$89 \%$} & \multicolumn{4}{|c|}{$80 \%$} & \multicolumn{4}{|c|}{$82 \%$} & \multicolumn{4}{|c|}{$86 \%$} & \multicolumn{4}{|c|}{$92 \%$} \\
\hline
\end{tabular}


was used. For the surveys, a combination of Survey 123 and ArcGIS StoryMaps was used. The vector tiles were hosted on ArcGIS Online and, the style variations were made in the online vector tile editor from ArcGIS (Esri, n.d.), which is available via ArcGIS Online. The vector tile version of the Esri World Topographic Map is available by default in the editor, while the raw data for the BRT Achtergrondkaart Standaard was hosted on ArcGIS online and could be downloaded. The style sheet, however, was provided by Kadaster and cannot be shared.

\section{Results}

The created methodology was applied to the selection of basemaps. For the CSA, the six most commonly used basemaps in the Netherlands have been assessed (Fig. 2 and Table 2). Fig 2 shows the different colors present in each map, from which pairs of colors could be created. The distinguishability of each pair has been

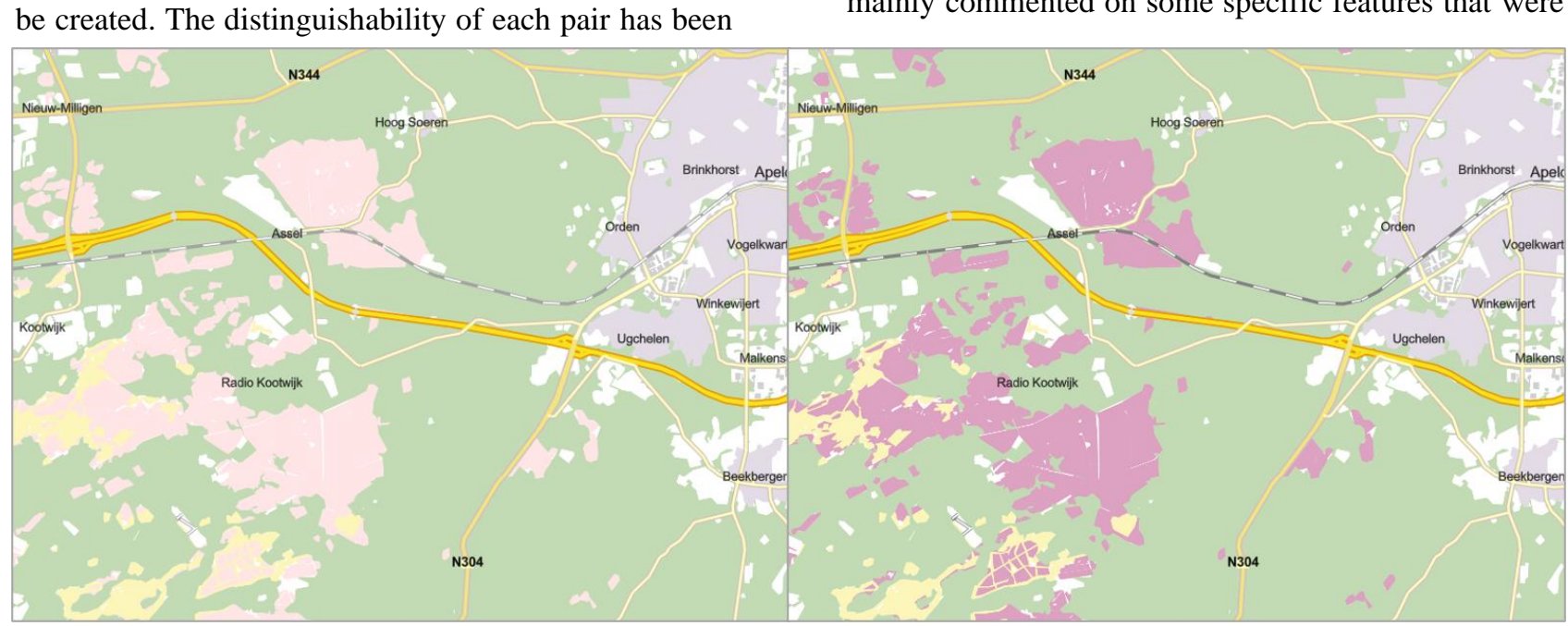

Figure 3. BRT Achtergrondkaart Standaard and improved version made for red, green, and blue color vision impairments.

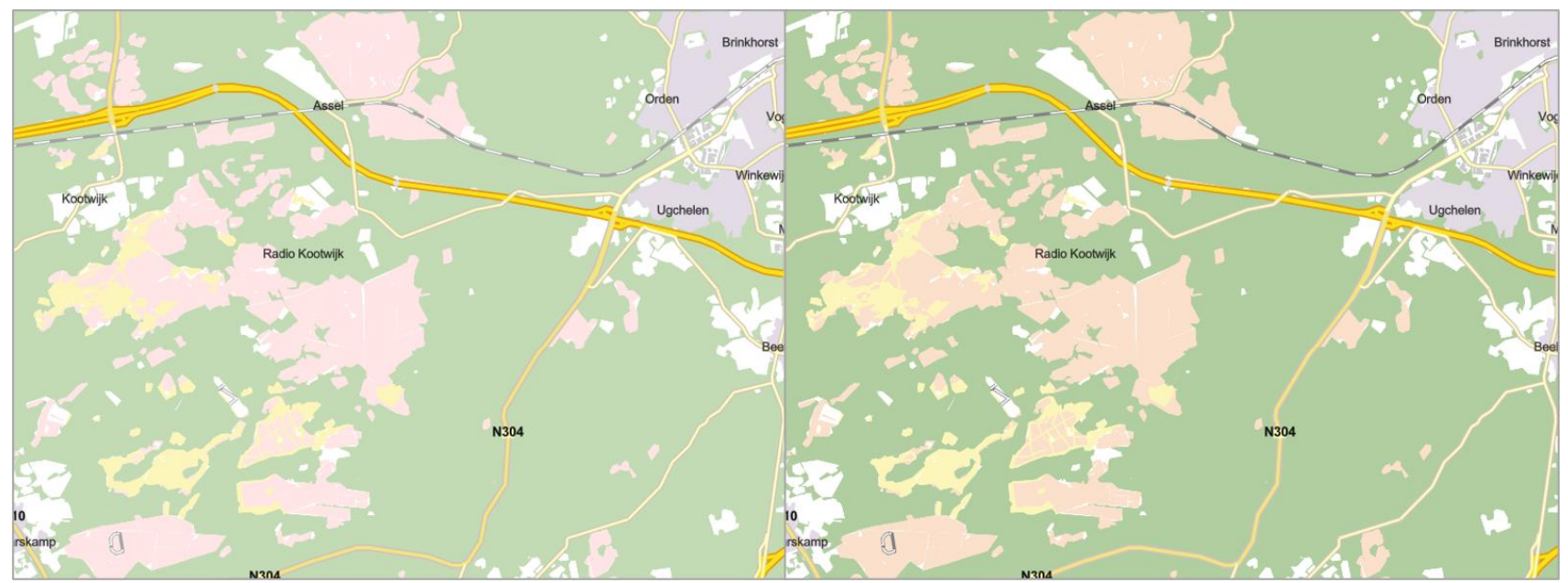

Figure 3. BRT Achtergrondkaart Standaard and second version of the improvement made for red, green, and blue color vision impairments. checked for every type of color vision impairment. Following that, the CSA table was filled in for all six basemaps (Table 2) with the information obtained with the accessement performed using the colorblind simulator. Both the grayscale basemaps, the Esri Light Gray Canvas and the BRT Achtergrondkaart Grijs, presented high scores as well as the Esri World Topographic Map. The other basemaps showed notable room for improvement in multiple areas.

A group of color vision impaired people was asked for feedback on the Esri World Topographic Map and BRT Achtergrondkaart Standaard. The feedback revealed that red and green color vision impaired people had the most trouble distinguishing the features on the basemap due to the color and contrast used for their representation. This was also because blue color vision impairment is rare and thus it was underrepresented in the survey. The respondents mainly commented on some specific features that were 


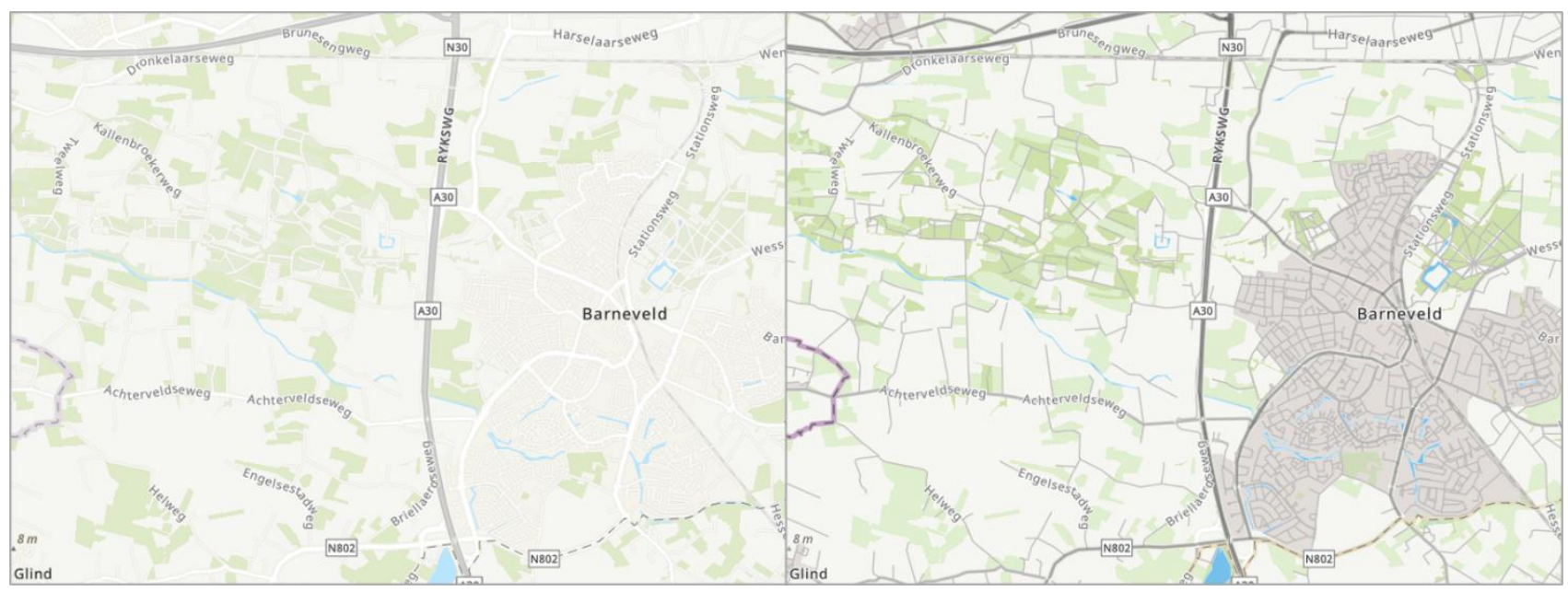

Figure 5. Esri world Topographic Map and improved version made for blue color vision impairment.

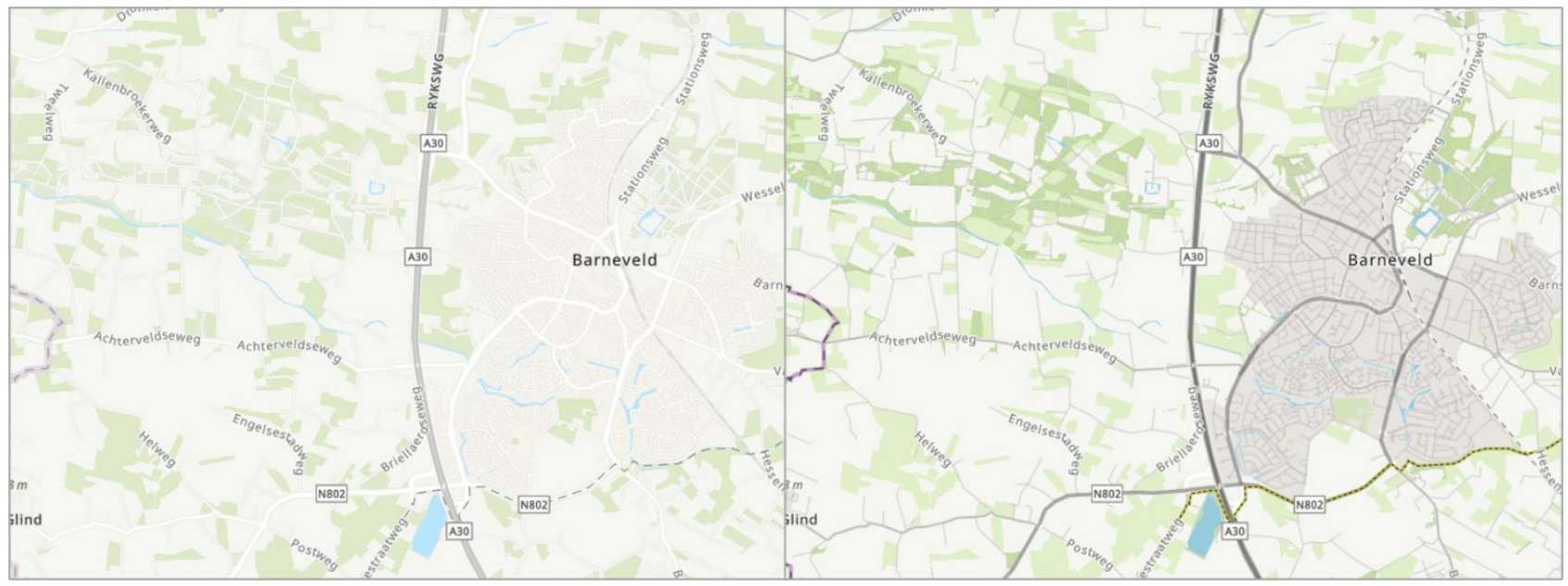

Figure 5. Esri world Topographic Map and improved version made for red and green color vision impairments.

not distinguishable from each other. In general, the BRT Achtergrondkaart Standaard was a better option for color vision impaired people than the Esri World Topographic Map.

Those two basemaps were further improved based on the initial feedback and the comments given after the first round of improvements. This led to the maps as illustrated in Fig. 3 to 6. For the BRT Achtergrondkaart Standaard (Fig 3 and 4), the improvements made for two different versions were minor and mostly focused on changing the color for heather, forests, railways, and buildings. This significantly improved the basemaps for all three types of color vision impairments. For the Esri World Topographic Map, two variations were created (Fig. 5 and 6), one for the blue type of color vision impairment and one for red and green color vision impairments. In both variations, the adjustments focused on changing the color for nature, roads, and urban areas. For nature, some features had to be generalized into one feature to limit the number of colors that had to be used.

Color vision impaired people who participated in the survey responded that the improvements made increased the distinguishability of the basemap features. However, for some users, the contrast between the different colors was still insufficient. Generally, the non-colorblind people preferred the original BRT Achtergrondkaart Standaard but appreciated the darker railroads on the improved basemap. For the Esri World Topographic Map, the non-colorblind people preferred the improved basemap over the original.

\section{Discussion}

The methods developed to evaluate the use of colors on the basemaps showed promising results. The evaluation performed using the colorblind simulator offered a good indication of problematic areas on the basemaps. 
The findings could be confirmed with the survey performed with color vision impaired people. However, assessing and editing complex maps with the eyedropper tool can be challenging due to the transparency added to some of the overlapping layers.

The evaluation of the BRT Achtergrondkaart Standard and the Esri World Topographic Map showed that for both basemaps some features were difficult to be distinguished due to the similarity of their colors. This result shows similarities to a study performed by (Kvitle, Pedersen, \& Nussbaum, 2016) with Norwegian maps which indicated that the quality of a map lies in the ability of the user to extract its features. The color selection needs to be chosen in a way that allows for the correct perception of those features. The perception of certain elements was a major issue because some of the features on the selected basemaps used the same lightness of color which leads to a reduction of contrast. This issue was better represented in the Esri World Topographic Map and could be adjusted by offering sufficient contrast between the colors, which according to (Peterson, 2015) is the solution to avoid problems for color vision impaired people, and can also be achieved by applying differences in brightness among the used colors.

The adjustment of colors performed in the BRT Achtergrondkaart Standard and the Esri World Topographic Map corresponds to the conclusions obtained by the work of (Jenny \& Vaughn Kelso, 2007). Their work suggests that colors with strong contrast should be used to avoid problematic combinations for color vision impaired people, and this corresponds to the approach used in the enhancements made in both basemaps.

After the improvements, the participants on the surveys offered valuable input of some of the colors chosen for certain features and the intensity with which they were represented. The inputs on the first improvements were essential for the follow-up round of enhancements in both basemaps. For the final optimization of the BRT Achtergrondkaart Standard and the Esri World Topographic Map, some colors were made darker, and others were softened in comparison to the initial adjustments. The administrative boundaries and the color of certain buildings were also adjusted on the Esri World Topographic Map to allow better identification of those features.

\section{Conclusion}

It can be concluded that research has already been done on the topic of optimizing the color use for color vision impaired people. These research though, have not been extensively applied to basemaps.

The main research question was answered by creating a method for improving basemaps for color vision impaired people. The method encompassed two main steps. In the first step, the current basemaps were assessed. The use of color was checked, and problematic color combinations were identified. The distinguishability of different map features was also assessed. The evaluation was finalized by checking the potentially problematic elements with actual color vision impaired people.

The next step was improving the basemaps in multiple iterations where each iteration ended with a survey among color vision impaired people. The responses of the survey indicated that the proposed method indeed led to significant improvements, even though further improvements are still required.

Based on the study, all basemaps could make use of a more colorblind-friendly version where it is advised the application the vector tile technology. Further research is advised into the effect of the optimized basemaps on the interpretation of overlay data. Additionally, the user research needs to be scaled up to create a more representative group of color vision impaired people.

\section{Acknowledgements}

This study was an initiative from Esri Nederland (Niels van der Vaart) and Kadaster (Vincent van Altena) as a collaboration with the ACT project of the GeoInformation Science master program at Wageningen University and Research. A StoryMap with more details of the project can be accessed at Tailoring basemaps for color vision impaired people (arcgis.com)

\section{References}

Colblindor. (2009, 03 15). Color Blind: Which Are Your Blind Colors? Retrieved 03 20, 2021, from Colblindors: https://www.colorblindness.com/2009/03/15/color-blind-whichare-your-blind-colors/ 
Colblindor. (2010). Color blind essentials. Retrieved 03 20, 2021, from https://www.colorblindness.com/2010/02/23/color-blindessentials/

Color Oracle. (2018, 05). Color Oracle: Design for the Color Impaired. (B. Jenny, Editor) Retrieved 03 20, 2021, from https://colororacle.org/

Esri. (2015, 10 27). Vector Basemaps. Retrieved 03 31, 2021 , from https://www.arcgis.com/home/overview

Esri. (n.d.). Vector tile style editor. Retrieved from https://developers.arcgis.com/vector-tile-styleeditor/

Harrower, M., \& Brewer, C. A. (2013, 07 08). ColorBrewer.org: An Online Tool for Selecting Colour Schemes for Maps. The Cartographic Journal, 27-37. doi:10.1179/000870403235002042

Hobbins, D. (2019). Map Design for the Color Vision Deficient. In Handbook of the Changing World Language Map (pp. 1-13). Springer. doi:https://doi.org/10.1007/978-3-319-734002_46-1

Jenny, B., \& Vaughn Kelso, N. (2007, 01). Designing Maps for the Colour-Vision Impaired. SoC BULLETIN Vol 41, 9 -12. doi:10.14714/CP58.270

Kadaster. (2020). Topographic map 1: 10,000. Retrieved 03 20, 2021, from Kadaster: https://www.kadaster.nl/producten/kaarten-en$\underline{\text { luchtfoto-s/actueel/topografische-kaarten }}$

Kvitle, A., Pedersen, M., \& Nussbaum, P. (2016, 02). Quality of color coding in maps for color deficient observers. Electronic Imaging, pp. 18.doi:10.2352/ISSN.2470-

1173.2016.20.COLOR-326

Okabe, M., \& Ito, K. (2002, 11 20). How to make figures and presentations that are friendly to color blind people. Retrieved from https://jfly.uni-koeln.de/html/color_blind/

Peterson, G. N. (2015). GIS Cartography A Guide to Effective Map Design (2nd ed.). Boca Raton: CRC Press Taylor and Francis Group.

Skinner, A. (2020, 10 22). Towards an Accessible Basemap. Retrieved from ArcGIS Blog: https://www.esri.com/arcgis-living- atlas/mapping/towards-an-accessible-

basemap/

Wong, B. (2011, 05 27). Points of view: Color blindness. Nature

Methods. 